Revue des patrimoines

\title{
L'Habitation Beauséjour : une plantation martiniquaise remarquable et pérenne
}

\author{
Laurent-Christian Ursulet
}

\section{(2) OpenEdition}

Journals

Édition électronique

URL : http://journals.openedition.org/insitu/2432

DOI : 10.4000/insitu.2432

ISSN : 1630-7305

Éditeur

Ministère de la culture

Référence électronique

Laurent-Christian Ursulet, «L'Habitation Beauséjour : une plantation martiniquaise remarquable et pérenne », In Situ [En ligne], 5 | 2004, mis en ligne le 19 avril 2012, consulté le 02 mai 2019. URL : http://journals.openedition.org/insitu/2432 ; DOI : 10.4000/insitu.2432

Ce document a été généré automatiquement le 2 mai 2019.

\section{(c) (i) (9)}

In Situ Revues des patrimoines est mis à disposition selon les termes de la licence Creative Commons Attribution - Pas d'Utilisation Commerciale - Pas de Modification 4.0 International. 


\title{
L'Habitation Beauséjour : une
} plantation martiniquaise remarquable et pérenne

\author{
Laurent-Christian Ursulet
}

«Les jumelles braquées sur cette côte nord de l'île
comblent instantanément la distance qui sépare la
perception commune du rêve des poètes. "
André Breton, Martinique. Charmeuse de serpent

En Martinique existe une « commune de l'extrême » dénommée Grand-Rivière, située à la limite nord de l'île. On y arrive par la « Porte de l'enfer », un chemin pentu et escarpé, adouci quelque 100 mètres plus bas par la Grande Rivière qui donne son nom à la commune. Sur les rives de celle-ci se dresse un bourg datant de la fin du XVII ${ }^{e}$ siècle cerné par une végétation dense et par la mer.

2 L'Habitation Beauséjour occupe une position méridionale dans cette géographie communale. Elle se situe un peu avant la « Porte de l'enfer », entre la rivière Potiche et la Grande Rivière, sur un plateau élevé à 102 mètres d'altitude au-dessus de l'anse Bagasse.

3 Miraculeusement épargnée par les ans, cette unité spatio-économique, architecturale et humaine conserve une structure et une infrastructure représentatives des "habitations », terme désignant à l'origine, aux Antilles françaises, des propriétés « d'habitants » (colons) destinées à une culture intensive d'exportation et possédant une main d'œuvre servile.

\section{Les origines de l'Habitation Beauséjour}

4 Le premier propriétaire de parcelles situées sur les terres de l'actuelle habitation fut Chambert Anthoine dit La Rivière, natif d'Agde dans le département de l'Hérault. 
Selon le Terrier de $1671^{2}$, qui est en fait un recueil de titres de concessions, la propriété mesurait alors 800 pas de large sur 1500 pas de haut ${ }^{3}$, soit une surface d'environ 150 hectares et s'étendait de la Rivière Potiche à la Grand'Rivière. Dotée d'une sucrerie et d'un moulin à bête, elle faisait partie des quelques cent neuf habitations sucrières traitant à l'époque les cannes d'un nombre à peu près égal d'habitations dépourvues de ces installations. En 1680, son effectif humain se composait du «maître de case », de sa femme, de ses deux enfants et de quatre-vingts nègres esclaves.

6 Ce domaine, dont la surface actuelle correspondrait en partie aux terres des anciennes habitations Malgré et Grand'Rivière, connut ensuite diverses mutations de propriété ainsi que de nombreux transferts de terrains, passant, du XVII ${ }^{e}$ au XXe siècle, entre les mains de neuf familles différentes : les Mirebeaux des Ruissaux, Desabayes, Levacher du Boullay, Brière, Potier de Courcy, de Chazaud, Ariès, Knight, et enfin les de Lucy de Fossarieu, propriétaires depuis 1928. Parmi toutes ces familles de colons originaires de France, seule la famille Knight était issue d'un patriarche de couleur martiniquais, Amédée Alexis Augustin Knight, ingénieur de l'Ecole Centrale de Paris, devenu sénateur de la Martinique en 1899.

Figure 1

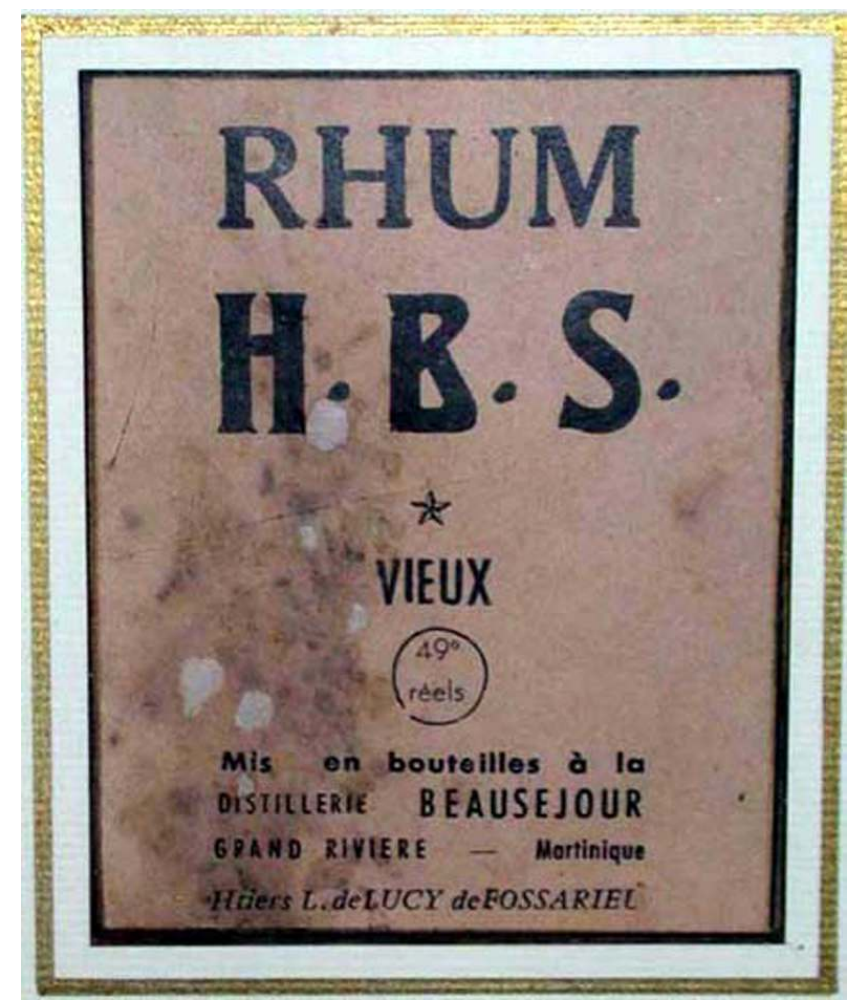

Etiquette du rhum HBS

Phot. Inv. L.-C. Ursulet @ Inventaire général, ADAGP, 2003

7 Planteur et distillateur à Saint-Pierre, il acquit cette terre au début du $\mathrm{XX}^{\mathrm{e}}$ siècle, dans un contexte de crise sucrière où les faillites d'habitations étaient monnaie courante. Malgré cela, l'exploitation connut sous sa direction une étonnante prospérité. Elle passa d'une surface de 136 hectares à 285 hectares et fut dotée d'une distillerie qui produisit un rhum de qualité, le « rhum H.B.S. » médaillé d'or à l'Exposition coloniale de Paris en 1932 (fig. $\mathbf{n}$ • 1). C'est également à cette époque, entre 1900 et 1912 que l'habitation prit le nom de 
Beauséjour. Quand les héritières d'Amédée Knight revendirent le domaine, en 1928, au « béké » Pierre François Honoré Louis de Lucy de Fossarieu, elle s'était encore agrandie de 50 hectares. On continuait à y cultiver la canne à sucre et à produire du rhum, tandis que le cacao ${ }^{4}$ déclinait au profit de cultures secondaires comme le maïs, les haricots ou la patate douce. Ce n'est qu'après la Seconde Guerre mondiale que le développement de la culture bananière supplanta définitivement les productions antérieures. Aujourd'hui encore, l'activité agricole de l'Habitation Beauséjour est consacrée à cette culture d'exportation.

\section{Ordonnance et permanence}

Figure 2

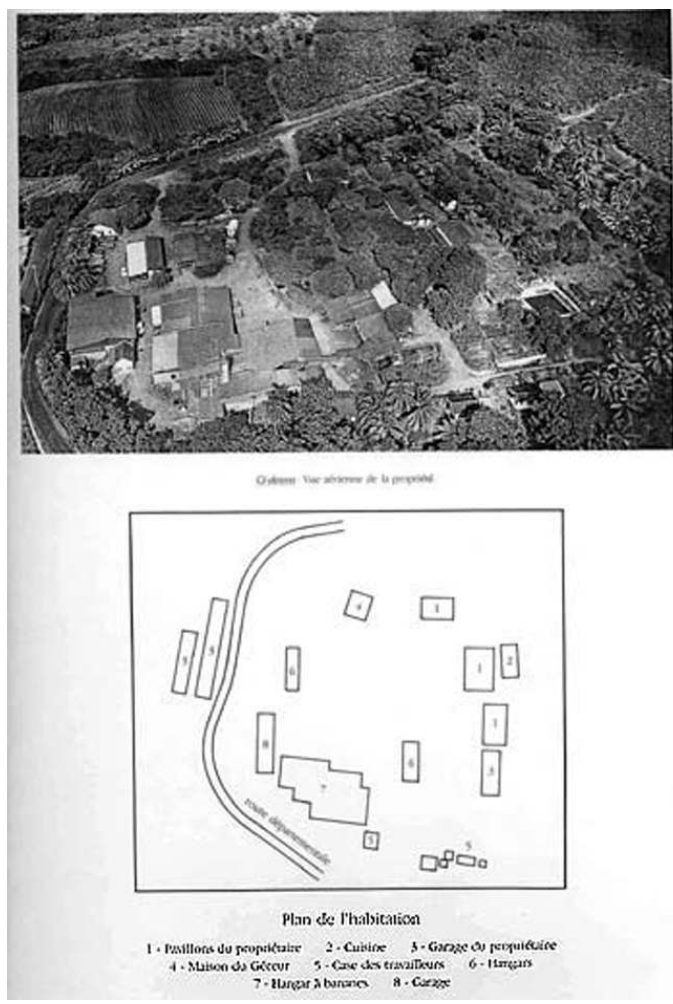

Vue aérienne et plan de l'Habitation Beauséjour

Phot. Inv. L.-C. Ursulet @ Inventaire général, ADAGP, 2003

8 La propriété s'ordonne en paliers successifs, de la mer jusqu'aux surfaces cultivées (fig. n - 2). Ses foyers concentriques sont formés par les trois maisons réservées aux propriétaires, implantées sur une terrasse dominant la zone industrielle, et par l'habitat ouvrier. La végétation y est très présente : vieux mahogany, palmiers royaux, touffes de bambou ou manguiers, et ouvre l'accès à la terrasse du jardin occupée par les logis. 
Figure 3

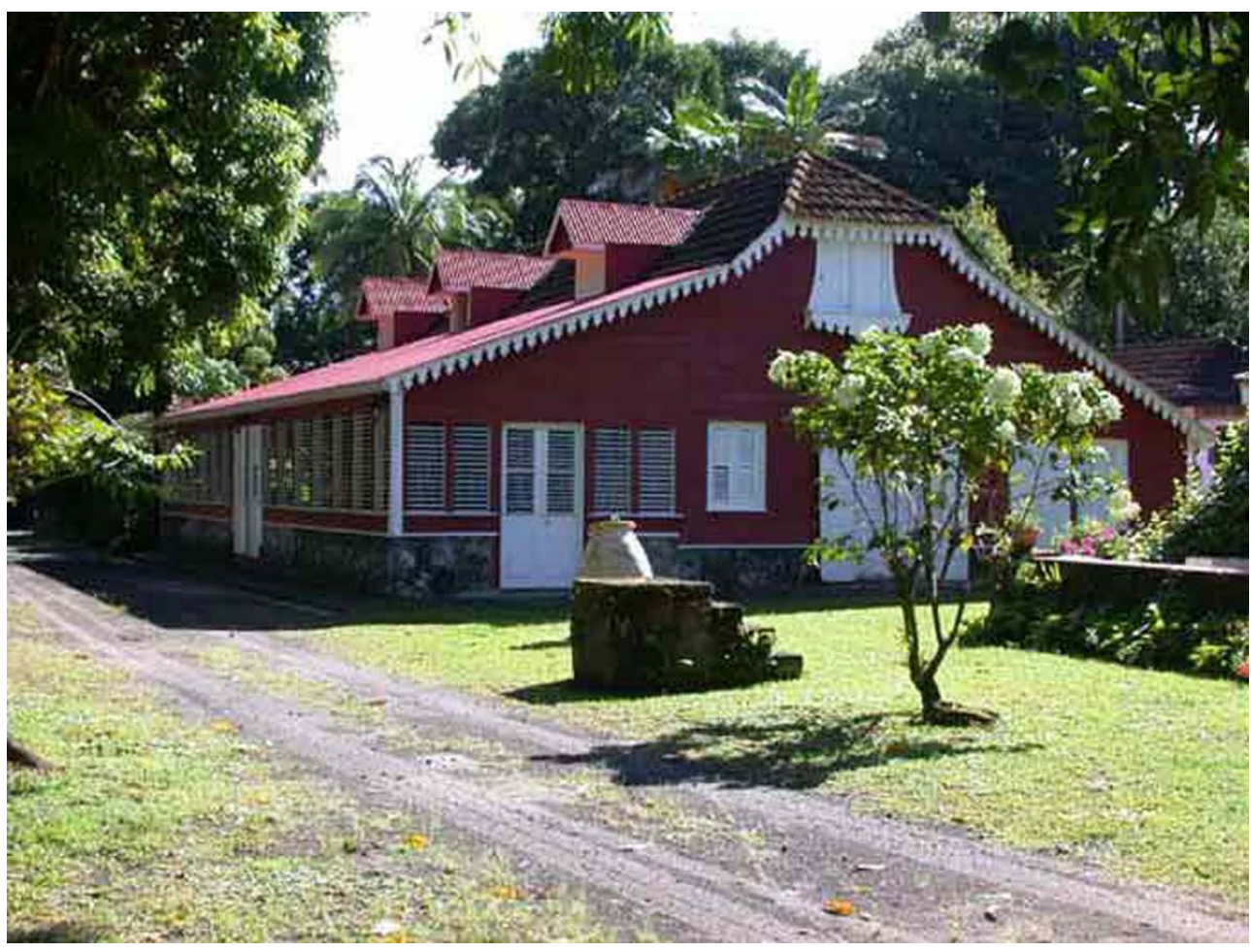

La maison de maître ou grand'case

Phot. Inv. L.-C. Ursulet ( Inventaire général, ADAGP, 2003

9 La «maison principale » (fig. $\mathbf{n}^{\circ} \mathbf{3}$ ), anciennement dénommée «maison de maître » ou "grand'case», est un beau témoignage du type de maison aujourd'hui appelée « coloniale ». Elle possède un corps de logis de plan rectangulaire, construit en « bois du Nord $»^{5}$, sur solin de $0,80 \mathrm{~m}$, en moellon sans chaîne couvert d'enduit et présentant un léger fruit. Une galerie fermée ${ }^{6}$, couverte en appentis, anime l'élévation antérieure. Le toit, à longs pans et demi-croupes, est couvert pour un tiers de tuiles mécaniques et pour le reste de tôles ondulées, sa forte pente facilite l'évacuation des eaux de pluie. L'aération du logis est efficacement assurée par des ouvertures percées en vis-à-vis dans les murs gouttereaux et les pignons, et renforcée par la présence de persiennes en bois.

Comme la plupart des autres habitations de l'île, l'édifice ne comporte aucun caractère monumental ou ostentatoire, son volume est équilibré par un rapport de proportions maîtrisé entre les murs et la toiture, sans artifice excessif. Quant au décor, il reste assez minimaliste, simplement composé de frises à lambrequins courant le long des élévations et d'impostes ajourées à décor géométrique ornant les baies intérieures.

Les deux autres maisons accueillent, pour l'une, les chambres à coucher des propriétaires actuels, pour l'autre, une école privée ainsi que des bureaux.

12 La première était à l'origine une dépendance de la maison principale. Initialement construite en rez-de-chaussée, elle servait autrefois de dépôt, de case à eau ${ }^{7}$ et d'écurie. Le linteau d'un encadrement en pierre de taille d'une baie du rez-de-chaussée possède encore une pierre gravée indiquant une probable date de construction : «12 AOUT 1811 ». En 1930, la bâtisse fut rehaussée d'un niveau en bois, avec une galerie bordée de colonnes. 
13 L'autre maison, appelée "pavillon du sénateur » parce que Monsieur Knight en fit un temps son logis, fut également construite durant la première moitié du XIXe siècle puis remaniée sur sa façade postérieure au début du $\mathrm{XX}^{\mathrm{e}}$ siècle. Aujourd'hui, c'est dans ses murs que se trouvent les bureaux des propriétaires et la petite école privée.

Cet ensemble, constitué des trois maisons principales, se caractérise donc par son architecture sobre mais distinguée, ses agréments décoratifs et son jardin, ainsi que par sa position géographique dominante au sein de l'habitation. Il marque, s'il en était besoin, la perpétuation d'une manière "d'habiter colonial», où la volonté d'éloigner les nuisances sonores ou olfactives inhérentes aux activités agricole et industrielle, était un souci majeur dans l'élaboration de l'infrastructure résidentielle du colon. Ainsi, se trouve$\mathrm{t}$-il placé en hauteur, derrière un mur de soutènement en pierre d'environ 2,60 $\mathrm{m}$ de hauteur, qui contient la poussée de la terrasse et fait aussi fonction de brise-vent.

\section{Habitation et industrie}

15 Au-delà de ce mur s'étend donc la sphère industrielle du domaine. On y trouve aujourd'hui une station de conditionnement dédiée à la banane ${ }^{8}$. C'est ici que l'on procède à « l'épistillage » puis au « dépattage » des fruits, qui permet de séparer les bananes de la hampe centrale. Ces dernières sont ensuite triées, calibrées, lavées, et conditionnées.

16 Toute l'activité se concentre au sein de cette unité industrielle, ensemble quelque peu dissymétrique de bâtiments desservis par de larges voies de manœuvre. Elle se compose d'une construction d'un peu moins de $1640 \mathrm{~m}^{2}$, originellement édifiée pour servir de sucrerie et reconstruite à la fin du XIX $\mathrm{X}^{\mathrm{e}}$ ou au début du XXe siècle à usage de distillerie. Elle présente aujourd'hui un rez-de-chaussée servant de magasin et de hangar d'emballage ainsi qu'un étage mansardé.

17 Sa façade est possède encore quelques moellons et bois d'origine habillant une structure métallique quasi intacte. Les apports d'aggloméré et de béton armé datant du début $\mathrm{XX}^{\mathrm{e}}$ siècle ainsi que des appentis en tôle ont fortement altéré l'harmonie architecturale et chromatique du bâti originel. Une cheminée quadrangulaire en pierre basaltique et brique recouverte partiellement d'enduit est adossée au mur-pignon gauche.

18 Tout autour s'élèvent les autres édifices : en contrebas de la terrasse d'habitation, un bâtiment de $15 \mathrm{~m} \mathrm{x} 4 \mathrm{~m}$, comprenant des bureaux, construit en partie en béton et en maçonnerie, avec menuiserie en bois et toiture en tôle nervurée, date du milieu $\mathrm{XX}^{\mathrm{e}}$ siècle. Du côté de la Route Nationale 1, un autre bâtiment de $14 \mathrm{~m} \mathrm{x} 15 \mathrm{~m}$, de structure métallique, datant de la première moitié $\mathrm{du} \mathrm{XX}$ siècle, abrite un atelier, un magasin ainsi qu'un réfectoire. En face du hangar de conditionnement de la banane on trouve un hangar métallique servant de garage et de magasin d'engrais. Un autre hangar fermé par des pans de tôle, disposé parallèlement à ce dernier, était appelé « charpenterie ».

\section{La gestion de l'eau}

19 L'industrie habitationnaire, essentiellement cannière jusqu'au milieu du XX $\mathrm{XX}^{\mathrm{e}}$ siècle, avait besoin d'une source d'énergie adéquate, d'où l'importance de l'énergie hydraulique et des ouvrages destinés à son utilisation sur le domaine. En effet, le problème de l'approvisionnement et du stockage de l'eau fut crucial lors de la colonisation. La survie de chacun, de même que l'exploitation profitable et durable des terres en dépendaient 
étroitement. Nombreux sont les ouvrages hydrauliques qui marquent aujourd'hui encore de leur empreinte le paysage de la Martinique (Canal de Beauregard, dit des Esclaves; Canal de Leyritz etc.)

20 A Beauséjour, outre la singulière roue hydraulique verticale d'un diamètre de $8,10 \mathrm{~m}$ et d'une largeur de 1,25, composée de dix bras doubles renforcés de croisillons en fer, fabriquée dans la première moitié du XIX siècle par la firme londonienne Fletcher \& Derby et située au rez-de-chaussée de l'ancienne distillerie devenue hangar à banane, l'élément remarquable est l'impressionnant aménagement hydraulique qui fut réalisé sur la propriété.

Figure 4

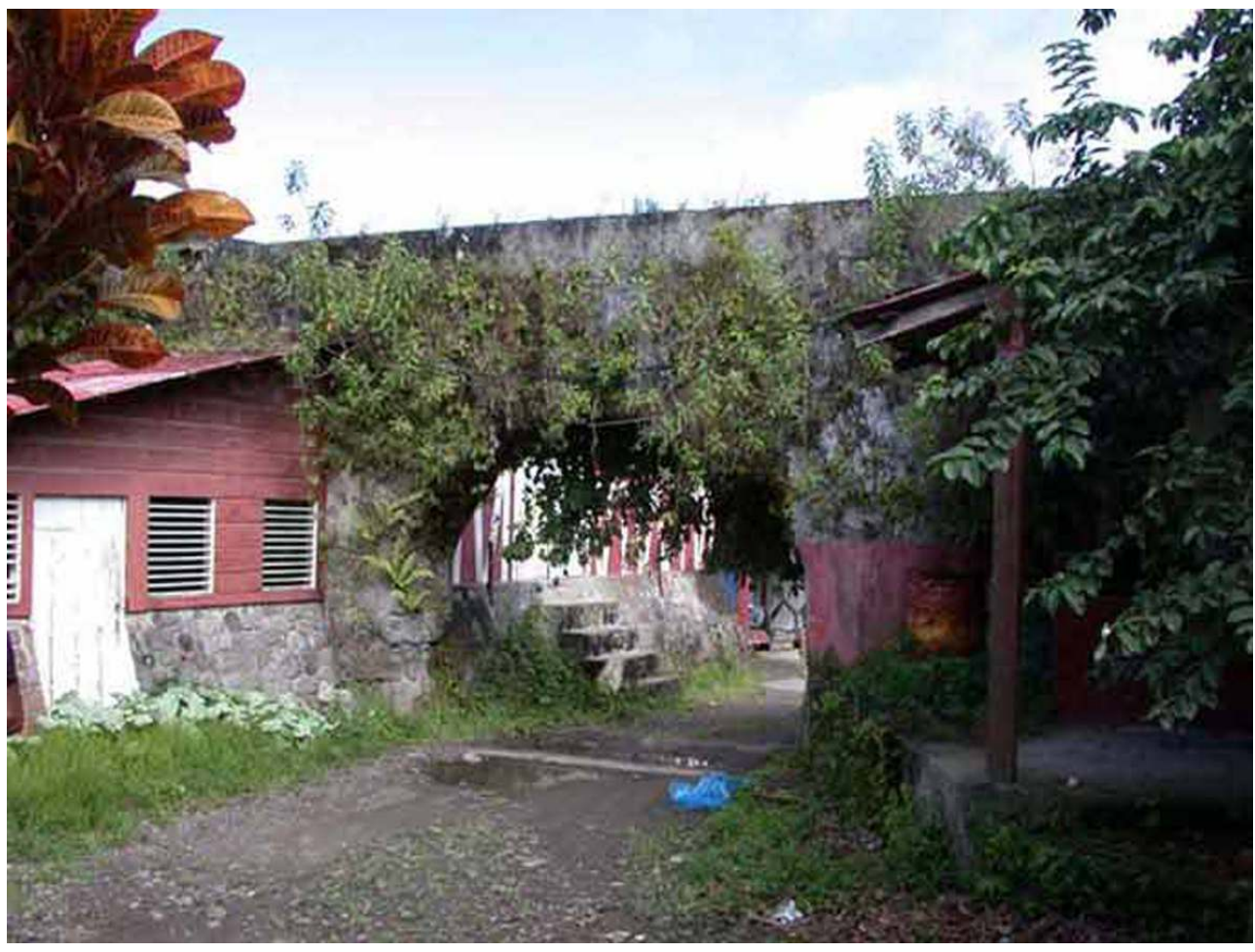

Aqueduc de l'Habitation Beauséjour

Phot. Inv. L.-C. Ursulet ( Inventaire général, ADAGP, 2003

Un canal, dit «canal de Beauséjour », fut construit par les esclaves entre 1800 et 1810 et servit à la fois à l'irrigation des plantations, au moulin hydraulique source d'énergie, et à l'alimentation en eau potable de l'Habitation par dérivation vers les deux bassins de la terrasse d'habitation principale. Il desservit un temps la distillerie d'une habitation voisine (Habitation Potiche) et s'étend aujourd'hui sur environ $4 \mathrm{~km}$. Sa prise d'eau se trouve sur la Grand'Rivière qu'il longe à flanc de paroi pour se trouver, en fin de parcours, côté habitation, à plus de $100 \mathrm{~m}$ de dénivelé par rapport à la rivière. Entièrement maçonné, il est actuellement envahi par la végétation et recouvert de terre ou d'éboulis mais a conservé plusieurs ouvrages d'art (fig. $\mathbf{n}^{\circ} 4$ ) qui permettent le franchissement des ravines ou des zones rocheuses dans lesquelles le canal n'a pu être creusé : dix aqueducs métalliques, en pierre et béton armé, un pont et deux tunnels. En 1979, lors du passage du cyclone David, deux aqueducs ont été détruits, et le canal rendu inutilisable malgré une remise en état de sa partie basse en 1988. 
En fin de parcours, le stockage des eaux était assuré par des bassins de retenue en maçonnerie situés contre le mur-pignon nord de la maison principale.

\section{Le logement des ouvriers}

Figure 5

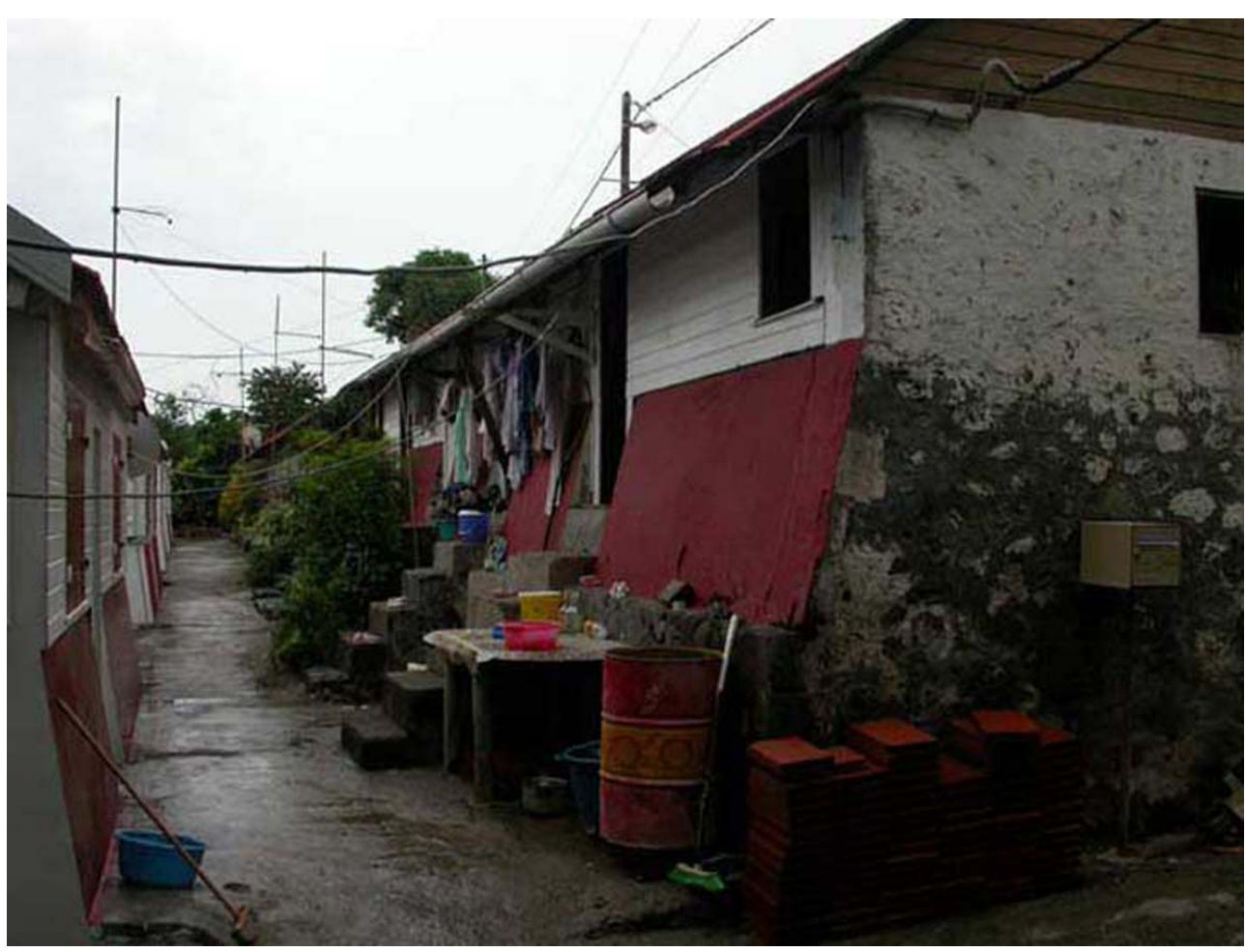

La « rue cases-nègres »

Phot. Inv. L.-C. Ursulet @ Inventaire général, ADAGP, 2003

La zone réservée au logement des ouvriers est constituée par ce que l'on a coutume d'appeler en Martinique une "rue cases-nègres" (fig. $\mathbf{n}^{\circ}$ 5). Bien que l'on trouve quelques logis d'employés en contrebas de la zone pavillonnaire, c'est à la limite septentrionale de l'Habitation, au nord de l'unité de conditionnement de la banane, après avoir traversé l'actuelle R.D. 10, que l'on accède à cette zone de baraquements destinés à accueillir les travailleurs agricoles. Datant de la fin du XVIII ${ }^{e}$ ou du début du XIX ${ }^{e}$ siècle, ils étaient réservés aux esclaves de l'exploitation.

Cette « rue cases-nègres » se compose de deux corps de bâtiments parallèles séparés par un espace formant rue, posés sur des solins talutés, d'environ $1 \mathrm{~m}$ de hauteur, en moellon recouvert d'enduit, avec des sols en béton. Les cloisons, les charpentes et les menuiseries sont en bois. Les toits à longs pans, dont l'un était autrefois couvert en tôle ondulée, l'autre en tuile mécanique, ont été récemment couverts en tuile (2003). Le premier bâtiment mesure une cinquantaine de mètres de longueur sur cinq mètres de large. Son élévation postérieure est aveugle et donne sur la route départementale. Il comprend six unités de logement qui possèdent chacune une cuisine extérieure en béton et ciment (anciennement simple appentis de tôle) située de l'autre côté d'une ruelle de moins de 2 
m la séparant des foyers. Le second bâtiment, en partie détruit par le cyclone de 1979, est moins long d'une vingtaine de mètres et s'organise comme le premier.

Ce type d'aménagement en baraque est minoritaire dans l'habitation martiniquaise, et se retrouve plus aisément dans le nord de l'Atlantique. A priori, il n'entre pas dans la catégorie des "rues cases-nègres", puisque aucune "case-nègre " ne s'y trouve. Cependant, il correspond parfaitement au type de logement d'esclave apparu au XVIII ${ }^{\mathrm{e}}$ siècle et caractérisé par des alignements de cases, proches les uns des autres, séparés par des ruelles. Ici, vient s'ajouter une dimension d'habitat-dortoir pensée durant la seconde moitié du XIX siècle afin de « caser » les travailleurs étrangers (Indiens, Chinois, Congos) amenés à la Martinique en appoint d'une main d'œuvre esclave devenue libre. Quant aux quelques cases de travailleurs présentes sur l'Habitation Beauséjour (construites à la même époque, puis restaurées durant la deuxième moitié du $\mathrm{XX}^{\mathrm{e}}$ siècle), elles sont réservées à un personnel jouissant d'un statut plus élevé que celui qui occupe les baraquements, et situées non loin de la zone des résidences principales. D'un confort nettement supérieur, elles se composent généralement de plusieurs chambres, d'une salle de bain, d'une cuisine, d'une galerie ouverte et bénéficient de l'eau courante.

\section{Habitation et beau séjour}

Figure 6

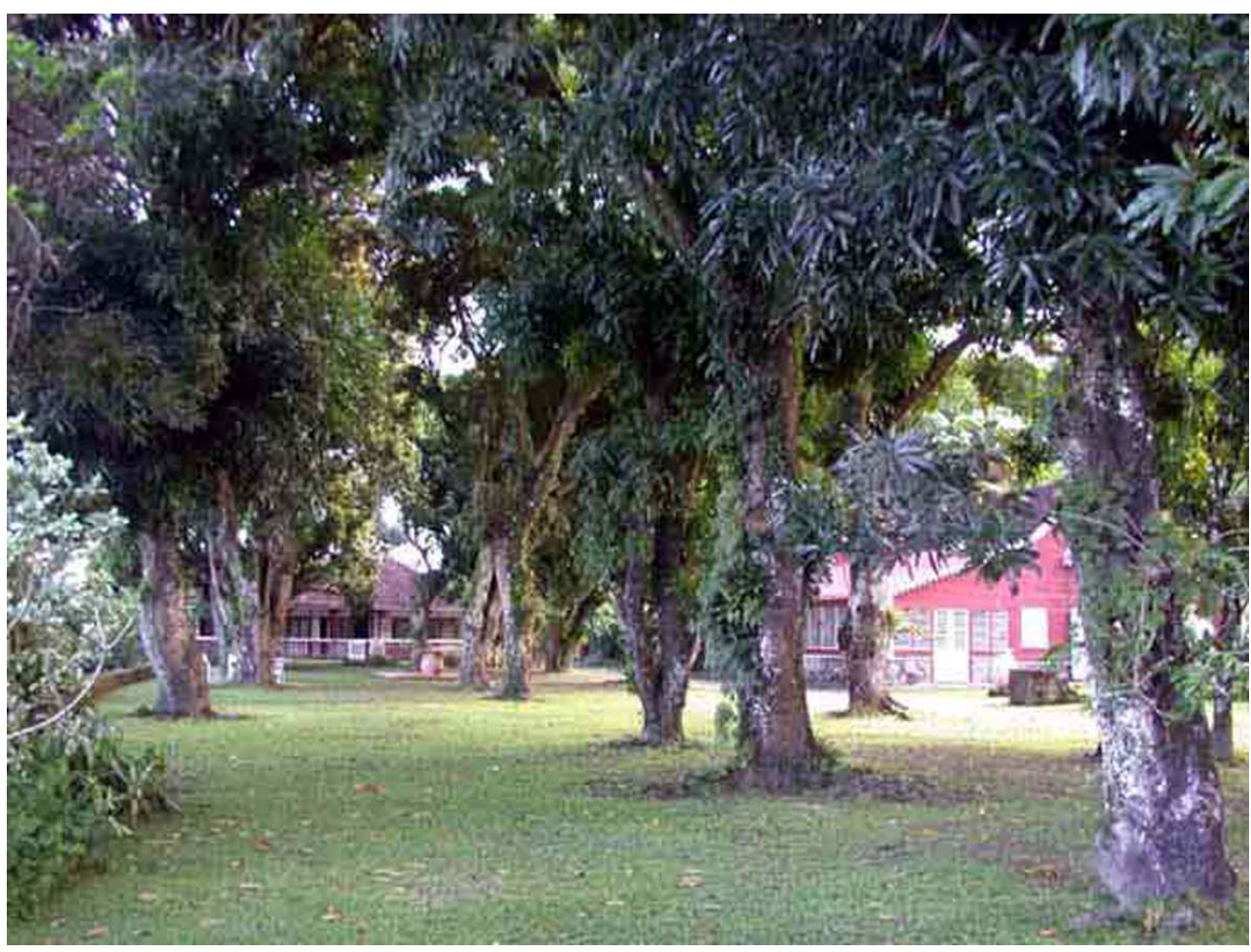

Terrasse du jardin plantée de manguiers

Phot. Inv. L.-C. Ursulet ( I Inventaire général, ADAGP, 2003

Enfin, il ne serait pas possible de conclure cette présentation de l'Habitation Beauséjour sans évoquer son locus amoenus, jardin d'agrément rare dans la région. Ses plans furent tracés en 1930 par Marie de Lucy de Fossarieu, épouse de Louis. Il comporte cinq terrasses successives présentant chacune un couvert différent. En parcourant celles-ci, on 
rencontre une grande variété d'essences (fig. $\mathbf{n}^{\circ}$ 6) (lianes de Jade, papyrus, fougères bleues, anthuriums, bougainvilliers, cannas jaune, fleurs de la passion, bambous, lataniers, bois d'Inde, orchidées, manguiers ou cocotiers), et des points de fraîcheur autour de bassins en pierre.

Un charme pittoresque auquel contribuent aussi quelques objets disposés en ces lieux au cours du temps, tels un cadran solaire (fig. $\mathbf{n}^{\circ} 7$ ) en pierre et marbre que l'on dit être le premier à avoir été introduit sur l'île, un montoir à cheval fabriqué pour la baronne de Courcy ou des jarres haïtiennes au galbe prononcé.

Figure 7

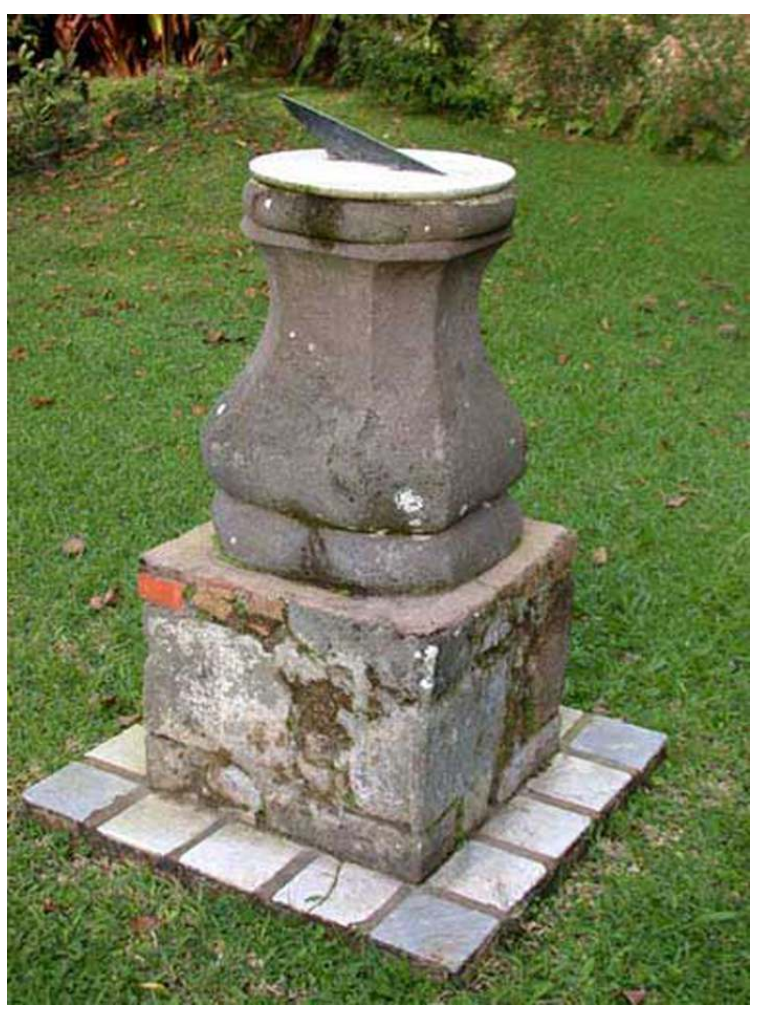

Cadran solaire dans le jardin

Phot. Inv. L.-C. Ursulet @ Inventaire général, ADAGP, 2003

Tout cet ordonnancement rappelle que, si à l'origine l'Habitation fut un lieu de travail servile entièrement dominé par des préoccupations liées à la production et à la rentabilité peu favorable au développement de formes architecturales qui ne seraient pas strictement utilitaires, la croissance de la richesse coloniale et donc de l'aisance des colons, puis l'abolition de l'esclavage entraînèrent une certaine recherche de confort et de beauté perceptible dans les constructions dédiées au logement des propriétaires et, dans une moindre mesure, de leurs ouvriers.

Malgré les diverses activités agricoles qui s'y sont succédé et les nombreux changements matériels opérés en son sein, la permanence de sa structure socio-spatiale et la récurrence des schémas de construction font de l'Habitation Beauséjour un cas d'espèce pour l'étude de ces micro-sociétés que constituent les plantations martiniquaises. Ces atouts architecturaux et anthropologiques en font un élément important du patrimoine rural de l'île, pour l'heure uniquement accessible au public lors des "Journées du patrimoine». 


\section{NOTES}

1. Ed. Jean-Jacques Pauvert, Paris, 1972.

2. Ou « Estat du procez verbal des terres de la Martinique ».

3. 1 pas équivalant à 3 pieds $1 / 2$, soit 1,134 mètres.

4. La dalle en terre cuite d'un ancien séchoir à cacao est encore présente derrière la maison de maître.

5. Bois importé d'Amérique possédant une sève résineuse éloignant les insectes.

6. La galerie était ouverte à l'origine et comprenait un bureau ainsi qu'un cabinet de toilette. Elle fut réaménagée durant les années 1930.

7. Selon l'historienne Mireille Mousnier, la case à eau, "création tout à fait originale de l'architecture créole » présentait à l'intérieur « des jarres alignées et installées dans un massif de maçonnerie montant jusqu'à leur col. » Ces jarres, d'environ $1 \mathrm{~m}$ de haut provenaient d'Aubagne, en Provence, et servaient à recueillir l'eau de pluie ou de l'eau de rivière purifiée avec un filtre de pierre volcanique. (Source: Mousnier, Mireille. De l'habitation sucrerie à l'usine : l'aménagement hydraulique aux Antilles françaises (XVII ${ }^{\mathrm{e}}-\mathrm{XX}^{\mathrm{e}}$ siècles). Actes du $\mathrm{III}^{\mathrm{e}}$ congrès national des Sociétés savantes, Poitiers, 1986.

8. Musa acuminata colla et Musa acuminata balbisiana.

9. Entité architecturale traditionnellement de trois mètres sur quatre ou de quatre sur six, avec toit à longs pans et solin de mur, composée de deux pièces et accueillant une cellule familiale.

\section{RÉSUMÉS}

Aux Antilles françaises les anciennes propriétés de colons ne se nomment pas plantations mais " habitations ", espace et mode de vie étant différents de ceux d'Amérique Latine, du Sud des Etats-Unis, ou des Grandes Antilles. L'Habitation Beauséjour, située sur des terres propices aux cultures d'exportation, dans le nord de la Martinique, est un exemple remarquable de domaine colonial qui a conservé une structure traditionnelle avec plantations, bâtiments principaux d'habitation, bâtiments de production, aménagement hydraulique, logements pour le personnel. $\mathrm{Au}$ cœur des rapports d'exploitation qu'engendrent les sociétés de type colonial, nous nous trouvons à Beauséjour devant une demeure créole dont la structure n'a pas varié de façon fondamentale au cours de son histoire.

In French West-Indies, the old colonial estates are not called plantations, but "habitations", because the geographic space as well as the way of life differed here from the vast plantations of Latin America, Southern United States and Greater Antilles. The "Habitation Beauséjour » situated on land that is good for growing export crops, in northern Martinique, is an instructive exemple of colonial estate preserving a traditional structure with plantations, main buildings, production buildings, hydraulic land-scaping, and buildings for housing and use of employees. The "Habitation Beauséjour" is more than many others a Creole estate with a persistent 
structure that has remained unchanged over its history and through the change due to the end of the exploitive relations generated by colonial societies.

INDEX

Mots-clés : demeure dite Habitation, maison Le Pavillon, maison de maître, maison Pavillon du sénateur, cité ouvrière dite rue cases-nègres, jardin d'agrément, distillerie, roue hydraulique, verticale, canal, sucrerie, cheminée, cuisine, bassin, aqueduc

\section{AUTEUR}

\section{LAURENT-CHRISTIAN URSULET}

Chercheur, Service régional de l'Inventaire, Direction régionale des affaires culturelles de Martinique. invite.martinique@culture.gouv.fr 